\title{
NARROW ABSORPTION COMPONENTS IN THE UV SPECTRA OF HD110432 (B1 IIIe)
}

\author{
Raman K. Prinja \\ Dept. of Physics \& Astronomy, UCL, Gower St., London, England. \\ Huib F. Henrichs \\ JILA, University of Colorado, Boulder, Colorado, U.S.A.
}

Introduction - We present high resolution $I U E$ ultraviolet observations of multiple narrow absorption components seen in the SiIII, NV, SiIV, and CIV profiles of the B1 Ille star, HD110432. Spectra taken during March 1986, spanning $\sim 11$ days, are modelled using line profile fits. Central velocities and column densities of the discrete features are derived.

The 11 observations obtained during March 1986 (SWP24923-28031) exhibit only minimal line profile variability, but are substantially different from the only other available IUE spectrum of HD110432, taken in April 1981 (fig. 1). This previous image reveals just a single extremely narrow (FWHM $\sim 50 \mathrm{~km} / \mathrm{s}$ ) feature at $\sim 1360 \mathrm{~km} / \mathrm{s}$.

Some examples of multiple discrete components in HD110432 are shown in fig. 2. The profiles are modelled assuming a constant 'underlying' P Cygni profile (derived from SWP13759), superimposed on which are the narrow features. We estimate for the stellar wind; $\dot{M} q_{i} \sim \mathbf{- 9 . 6 7},-9.55,-9.03,-9.67$ dex for Silll, $\mathrm{NV}, \mathrm{Si}$ IV, and C IV respectively $\left(\dot{M}\right.$ is the mass-loss rate in $M_{\odot} y r^{-1}$, and $q_{i}$ is the wind ion fraction).

The observed velocities of the narrow absorption components are illustrated in fig. 3. Up to 3 components may be present, though two principal features are consistently identiffed at $\sim-400 \mathrm{~km} / \mathrm{s}$ and $\sim-1100 \mathrm{~km} / \mathrm{s}$.

The narrow component ion fraction is plotted in fig. 4, over all the spectra in March 1986, as a function of velocity. The ion ratios may be correlated with the velocities of the discrete components.
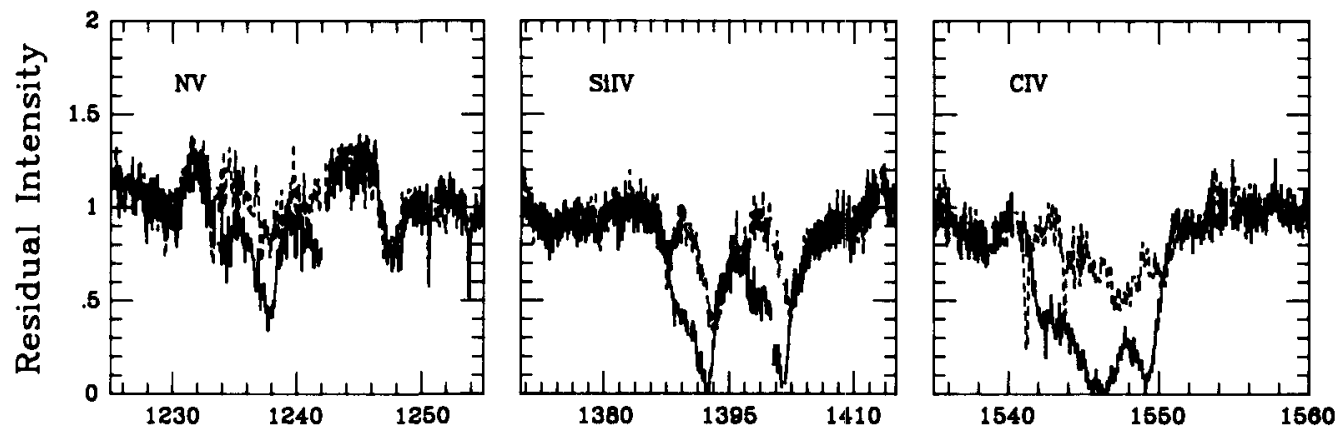

Wavelength $(\AA)$

FIG. 1 - Extensive narrow component variability is apparent between the observation in April 1981 (dotted line; SWP13759) and the typical (more enhanced) opectra of March 1886 (oolid line; e.g. SWP27928). 


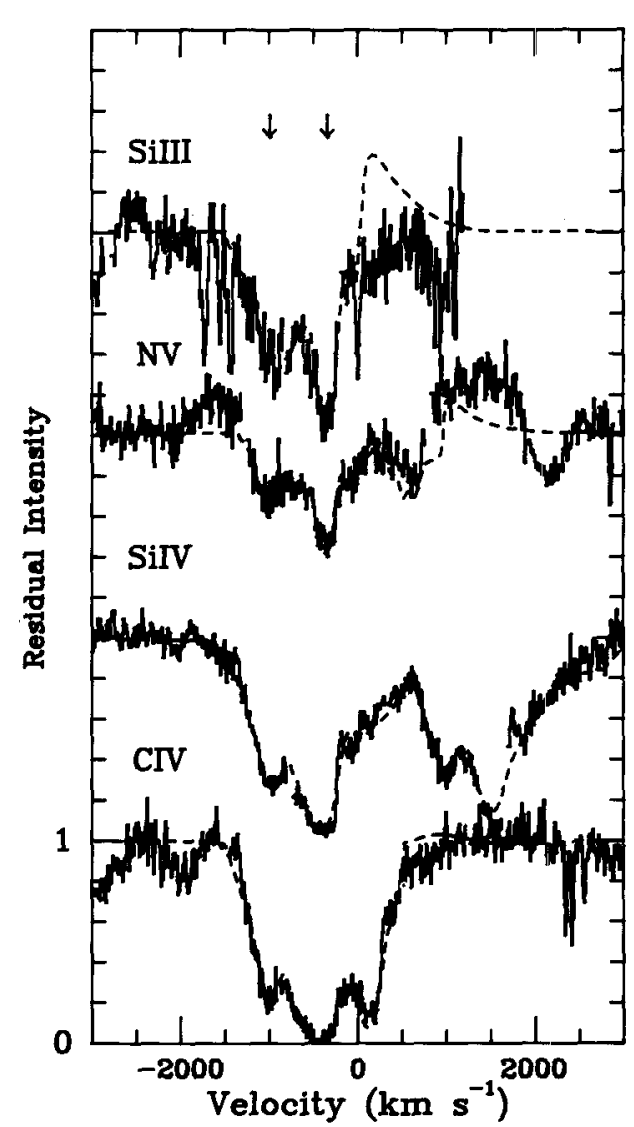

FIG. 2 - Some examples of UV line profiles in HD110492. Dashed lines are the adopted model fits. Mean narrow component positions are indicated.

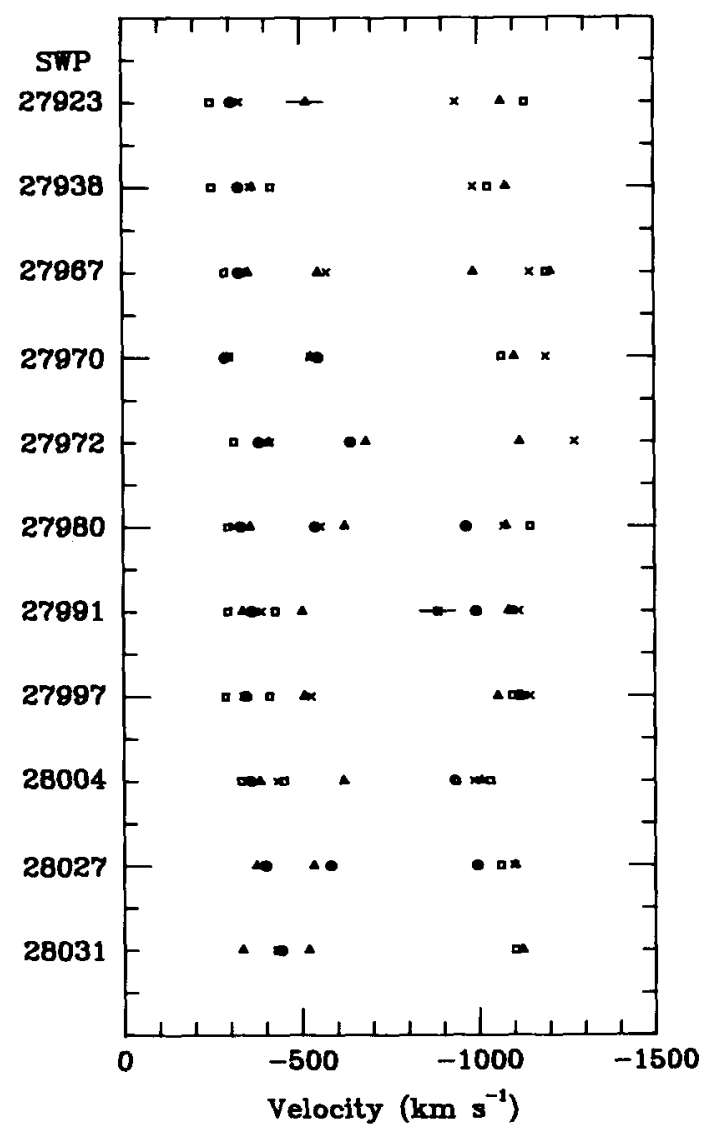

FIG. 3 - The narrow component velocities observed in $\operatorname{SiIII}(\bullet), N V$ (), $\operatorname{Si} I V(\times)$, and $C I V(\Delta)$.

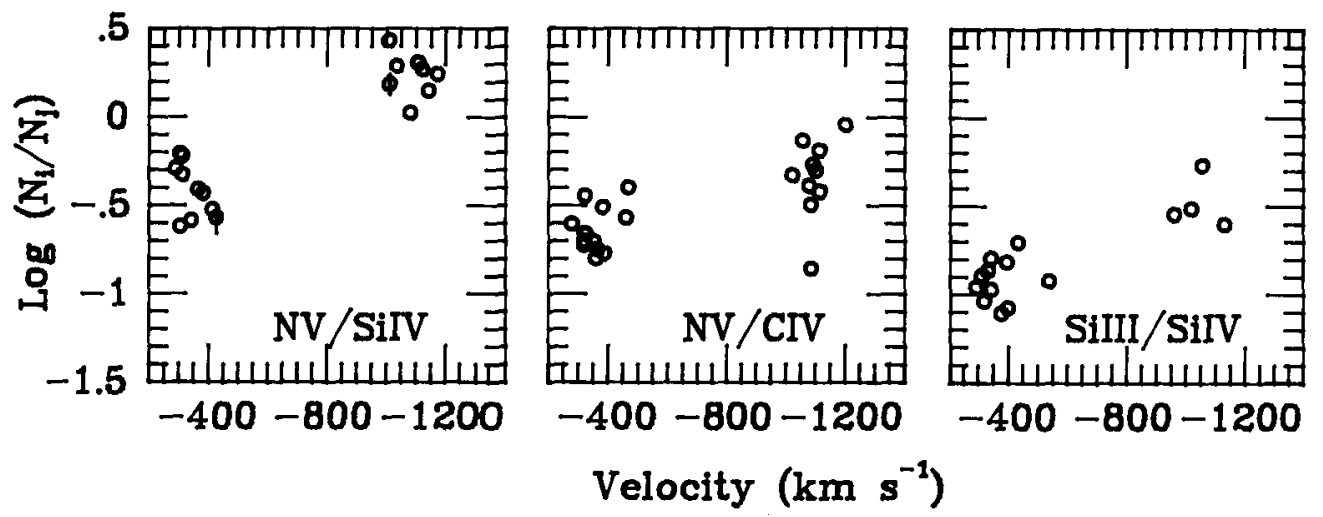

FIG. 4 - The ion fraction ratios observed in the narrow components as a function of velocity. 


\section{DISCUSSION FOLLOWING PRINJA}

Henrichs:

It would be interesting to see if simultaneous IR observations exist. We heard two reports earlier today on this star.

Lamers:

Your observed increase in the degree of ionization in the narrow components agrees with two facts observed in normal stars: (1) The narrow components at about $0.7 v_{\infty}$ observed in normal stars have a higher degree of ionization than the normal winds (Lamers et al., 1982). (2) I recently found evidence for blobs ejected from $\alpha$ Cam (09.5 Ia) which change to higher ionization as they move out (Lamers et al., 1986).

Underhill:

Did you have to introduce a large microturbulence of the order of $100 \mathrm{kms}^{-1}$ to fit your discrete components?

Prinja:

No, it was not necessary. 